\title{
High glucose mediates endothelial-to-chondrocyte transition in human aortic endothelial cells
}

\author{
Rining Tang, Min Gao, Min Wu, Hong Liu, Xiaoliang Zhang and Bicheng Liu*
}

\begin{abstract}
Background: Vascular calcification is one of the common complications in diabetes mellitus. Many studies have shown that high glucose $(\mathrm{HG})$ caused cardiovascular calcification, but its underlying mechanism is not fully understood. Recently, medial calcification has been most commonly described in the vessels of patients with diabetes. Chondrocytes were involved in the medial calcification. Recent studies have shown that the conversion into mesenchymal stem cells (MSCs) via the endothelial-to-mesenchymal transition (EndMT) could be triggered in chondrocytes. Our previous research has indicated that HG induced EndMT in human aortic endothelial cells (HAECS). Therefore, we addressed the question of whether HG-induced EndMT could be transitioned into MSCs and differentiated into chondrocytes.
\end{abstract}

Methods: HAECs were divided into three groups: a normal glucose (NG) group, HG group (30 mmol/L), and mannitol $(5.5 \mathrm{mmol} / \mathrm{L} \mathrm{NG}+24.5 \mathrm{mmol} / \mathrm{L})$ group. Pathological changes were investigated using fluorescence microscopy and electron microscopy. Immunofluorescence staining was performed to detect the co-expression of endothelial markers, such as CD31, and fibroblast markers, such as fibroblast-specific protein 1 (FSP-1). The expression of FSP-1 was detected by real time-PCR and western blots. Endothelial-derived MSCs were grown in MSC medium for one week. The expression of the MSCs markers STRO-1, CD44, CD10 and the chondrocyte marker SOX9 was detected by immunofluorescence staining and western blots. Chondrocyte expression was detected by alcian blue staining. Calcium deposits were analyzed by alizarin red staining.

Results: The incubation of HAECs exposed to HG resulted in a fibroblast-like phenotype. Double staining of the HAECs indicated a co-localization of CD31 and FSP-1. The expression of FSP-1 was significantly increased in the HG group, and the cells undergoing EndMT also expressed STRO-1, CD44 and SOX9 compared with the controls $(P<0.05)$. Additionally, alcian blue staining in the HG group was positive compared to the NG group. Consistent with the evaluation of SOX9 expression, calcium deposits analyzed by alizarin red staining were also enhanced by the HG treatment. Specifically, we showed that HG-induced EndMT is accompanied by the activation of the canonical Snail pathway.

Conclusions: Our study demonstrated that HG could induce endothelial cells transdifferentiation into chondrocyte-like cells via the EndMT, which is mediated in part by the activation of the Snail signaling pathway.

Keywords: High glucose, Vascular calcification, Endothelial-to-mesenchymal transition, Mesenchymal stem cells, Snail

\footnotetext{
*Correspondence: liubc64@yahoo.com.cn

Institute of Nephrology, ZhongDa Hospital, school of medicine, Southeast University, 210009 Nanjing, China
}

\section{() Biomed Central}

(c) 2012 Tang et al.; licensee BioMed Central Ltd. This is an Open Access article distributed under the terms of the Creative Commons Attribution License (http://creativecommons.org/licenses/by/2.0), which permits unrestricted use, distribution, and reproduction in any medium, provided the original work is properly cited. 


\section{Background}

Cardiovascular complications are the leading cause of death in patients with diabetes mellitus (DM) [1]. Emerging evidence suggests that the presence of vascular calcification in any arterial wall in patients with DM is associated with a 3-4-fold higher risk for mortality and cardiovascular events [2]. Pathological studies have shown that patients with DM exhibit characteristic calcification in the tunica media, which is independently associated with cardiovascular mortality [3]. However, the pathogenesis of medial artery calcification (MAC) is complex and has not been fully elucidated.

Traditionally, a calcium-phosphorus homeostasis imbalance is considered the cause of calcium salt deposition, which may play an important role in vascular calcification by transforming vascular smooth muscle cells (SMCs) to osteoblast-like cells, producing a matrix of bone collagen and non-collagenous proteins. MAC has recently been considered an orchestrated process that begins with mesenchymal condensation, followed by chondrogenesis and endochondral ossification [4,5]. However, the mechanisms responsible for these processes in diabetic vascular calcification remain largely unknown.

In the past decade, SMCs and pericytes have been reported to possess the plasticity to express bone and cartilage proteins in calcified blood vessels [6,7]. However, recent studies have indicated that mature endothelial cells can transform into fibroblasts in vitro and in DM [8-11], by a process known as the endothelialmesenchymal transition (EndMT). Furthermore, Medici et al. found that vascular endothelial cells have the potential to convert into mesenchymal cells that possess mesenchymal stem cells (MSCs) properties and are able to differentiate into chondrocytes [12]. In addition, chondrocyte conversion underlies medial calcification in uremic rats [13]. Thus, whether EndMT might be a novel source of chondrocytes during the MAC process in patients with DM is an interesting question.
Chronic high glucose (HG) is a major initiator of diabetic vascular complications and implicated in the development of vascular calcification [14,15]. Our previous studies have shown that $30 \mathrm{mmol} / \mathrm{L} \mathrm{HG}$ induced human aortic endothelial damage via the mediation of EndMT and that EndMT contributed to cardiac fibrosis in diabetic rats, which was inhibited by irbesartan [16,17]. However, the relationship between HG-induced EndMT and its association with chondrocyte transformation during diabetic vascular calcification are still poorly understood. In this study, we addressed the question of whether HG-induced EndMT could be used to transition human aortic endothelial cells (HAECs) into MSCs and then differentiate into chondrocytes.

\section{Materials and methods \\ Cell culture}

Primary HAECs were purchased from Sciencell Research Laboratories (USA) and cultured as previously described [16]. Briefly, cells were grown in endothelial culture medium (No. 1001, Sciencell) containing 5\% fetal bovine serum (FBS) (No. 0025), 1\% endothelial cell growth supplement (No. 1052) and 1\% penicillin/streptomycin solution (No. 0503) in $5 \% \mathrm{CO}_{2}$ at $37^{\circ} \mathrm{C}$. Passage 2-5 HAECs were expanded in monolayers in flasks or dishes. At approximately $80 \%$ confluence, the culture medium was changed to a serum-free solution for $24 \mathrm{~h}$ prior to their use in all experiments. And the HAECs were treated with normal glucose (NG; $5.5 \mathrm{mmol} / \mathrm{L}), \mathrm{HG}(30 \mathrm{mmol} / \mathrm{L}$ D-glucose) [16], or $5.5 \mathrm{mmol} / \mathrm{L} \mathrm{NG}+24.5 \mathrm{mmol} / \mathrm{L}$ mannitol for $48 \mathrm{~h}$.

\section{Cell differentiation}

The cells were grown in chondrogenic differentiation medium (MCDM, Sciencell) supplemented with TGF- $\beta 3$ (Peprotech, Rocky Hill, USA) at a concentration of $10 \mathrm{ng} / \mathrm{ml}$, followed by growth in serum-free medium with $\mathrm{HG}$ at $30 \mathrm{mmol} / \mathrm{L}$ for $48 \mathrm{~h}$. Alcian blue (Sigma) staining for chondrogenic proteoglycans and alizarin red

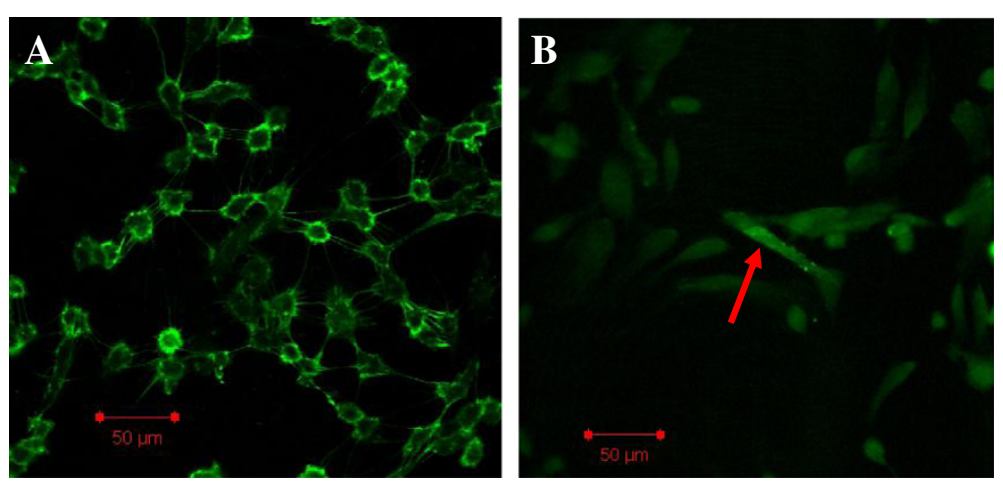

Figure 1 Immunofluorescence staining of HAECs with CD31 in the NG and HG groups. Note: The incubation of HAECS with high glucose (30 mmol/L) for $48 \mathrm{~h}$ resulted in a fibroblast-like phenotype (B). $1 \mathrm{bar}=50 \mu \mathrm{m}$. A: normal glucose. B: high glucose. 

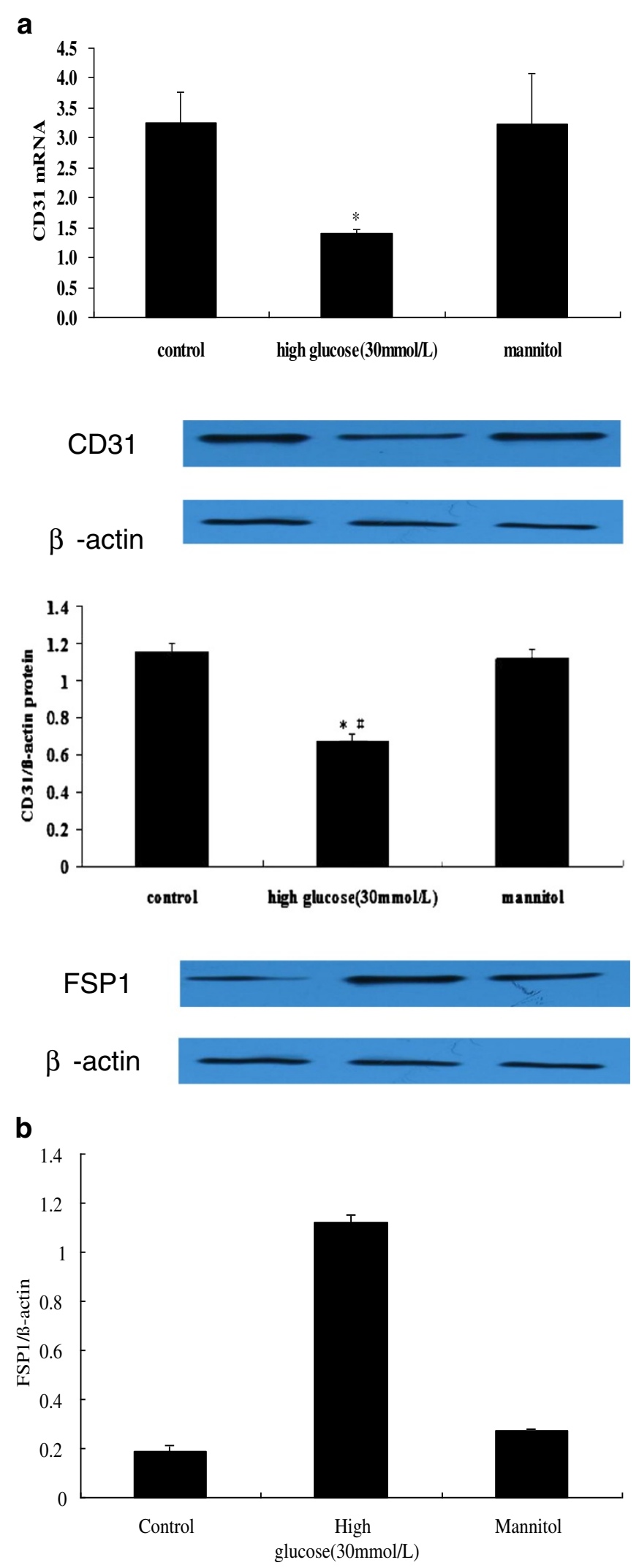

Figure 2 (See legend on next page.) 
(See figure on previous page.)

Figure 2 RT-PCR and western blot expression of CD31 in the NG and HG groups. Notes: The mRNA and protein expression of CD31 were decreased in cells incubated with high glucose, whereas the expression of FSP-1 was increased. ${ }^{*} P<0.05$ vs. control; \# $P<0.05$ vs. mannitol.

staining for calcium deposition were performed on cultures grown in chondrogenic medium for 7 days.

\section{Real-time PCR}

The total RNA from the cultured HAECs was extracted using RNAiso Plus according to the manufacturer's protocol (TAKARA, China). The RNA concentration and purity were confirmed with a Nanodrop 2000 (Thermo, USA). Samples with a relative absorbance ratio between 1.8 and 2.0 at 260/280 were used. All RNA samples were reverse transcribed (Applied Biosystems, USA).

The quantification of specific mRNAs was conducted using an ABI Prism 7300 Sequence Detection System (Applied Biosystems, USA) with the SYBR Green Real-time PCR Kit (TAKARA, China). The following oligonucleotide primer sequences were used: CD31, forward 5'-GAGTCCAGCCGCATATCC-3' and reverse 5'-TGACACAATCGTATCTTCCTTC-3'; FSP1, forward 5'- GTCCACCTTCCACAAGTAC-3' ${ }^{\prime}$ and reverse $5^{\prime}$ TG TCCAAGTTGCTCATCAG-3; CD44, forward 5'-GAG CAGCACTTCAGGAGGTTAC- $3^{\prime}$ and reverse $5^{\prime}$-GGAA TGTGTCTTGGTCTCTGGTAG-3'; CD10, forward $5^{\prime}$-C CTCGTTGACTGGTGGACTC-3' and reverse $5^{\prime}$-CTG ATAGGCTCTGTATGCTTGAC-3'; SOX9, forward $5^{\prime}-\mathrm{G}$ CTCTGGAGACTTCTGAAC-3' and reverse $5^{\prime}$-CGTT CTTCACCGACTTCC-3'; and $\beta$-actin, forward $5^{\prime}$-CTGG AAGGTGGACAGCGAGG-3' ${ }^{\prime}$ and reverse $5^{\prime}$-TGACGTG GACATCCGCAAAG- ${ }^{\prime}$. All primers were designed and synthesized by Generay (Shanghai). The relative amount of mRNA was normalized to $\beta$-actin and calculated using the standard curve method. In brief, the pre-PCR product of each gene was used as the standard. The standard curve was established with a 10-fold serial dilution of the product and was included in all PCR runs. The ratio of target gene abundance to housekeeping gene abundance was used to evaluate the expression level of each gene. Controls consisting of $\mathrm{ddH}_{2} \mathrm{O}$ were negative in all runs.

\section{Western blot analysis}

The total cellular protein was extracted to evaluate the levels of CD31, FSP1, $\alpha$-SMA, CD44, STRO-1 and CD10. Equal amounts of cell lysate proteins $(30 \mu \mathrm{g})$ were separated on 4-20\% SDS-polyacrylamide gels and transferred onto nitrocellulose membranes (Pall, USA) by electroblotting. The blots were incubated overnight with primary antibodies at concentrations recommended by the respective manufacturers: CD31 (sc-65260, Santa Cruz), FSP1 (ab27957, Abcam), CD44 (sc-71220, Santa Cruz), and CD10 (sc-9149, Santa Cruz). A horse-radish peroxidase-labeled secondary IgG (Santa Cruz, Europe) was then added to the blots. The signals were detected using an ECL advance system (GE Healthcare, UK). $\beta$-actin was used as the internal control.

\section{Immunofluorescence}

HAECs grown on coverslips were fixed in $4 \%$ paraformaldehyde and permeabilized with $0.3 \%$ Trition-X100. After blocking with $10 \%$ BSA for $1 \mathrm{~h}$, they were incubated with primary antibodies at $4^{\circ} \mathrm{C}$ overnight. The cells were then incubated with AlexaFluor-conjugated secondary antibodies (Invitrogen Technology, USA) at room temperature in the dark for $1 \mathrm{~h}$. The absence of primary antibody was used as a negative control. The cells were visualized and photographed with a scanning
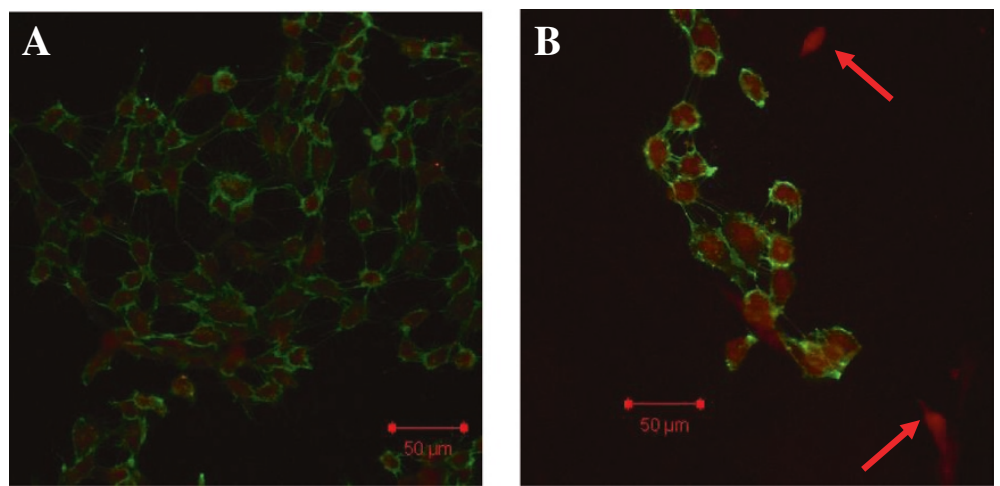

Figure 3 The confocal microscopy analysis. Notes: Labeling experiments used antibodies against CD31 (endothelial cell marker; green) and the fibroblast marker FSP1 (red). Confocal microscopy revealed that some cells acquired FSP1 staining and lost CD31 staining, which suggested the stage of EndMT. A: normal glucose. B: high glucose. 
confocal microscope (LSM 510 META, Carl Zeiss, Germany; TCS SP5, Leica, Germany).

\section{Electron microscopy}

Ultra-thin cells were counter-stained with uranyl acetate and lead citrate and were examined with a transmission electron microscope (TEM, HITACHI H600). Dried samples were sputtered with gold for observation by a scanning electron microscope (SEM-505, Phillips, the Netherlands).

\section{Statistical analysis}

The data were expressed as the means \pm standard deviation (SD). A one-way analysis of variance (ANOVA) was performed and confirmed with a two-tailed paired Student's $t$ test using SPSS 19.0. $P$ values less than 0.5 were considered significant.

\section{Results}

\section{HG induces EndMT in HAECs}

As our previous experiment showed, we found that HAECs treated with $30 \mathrm{mmol} / \mathrm{L} \mathrm{HG}$ for $48 \mathrm{~h}$ induced profound changes, with the cells becoming elongated, spindle-shaped and losing cobblestone morphology under fluorescence microscopy (Figure 1). The protein and mRNA expressions of endothelial marker CD31 were decreased in the cells incubated with HG, whereas the protein expression of FSP-1 was increased (Figure 2). Immunofluorescence with antibodies for the endothelial marker and the mesenchymal marker demonstrated that the HG-treated cells acquired FSP1 staining and lost CD31 staining compared with the control cells (Figure 3).

We next observed the cells under TEM and SEM. HAECs treated with $30 \mathrm{mmol} / \mathrm{L}$ HG for $48 \mathrm{~h}$ showed a distinct change from a cobblestone-like to a spindleshaped morphology. In addition, TEM was performed to examine the ultrastructure of cells, which displayed normal structures. In contrast, the HG group treated for $48 \mathrm{~h}$ exhibited endothelial protrusion, a significantly roughened endoplasmic reticulum, and microfilamentation (Figure 4). These data suggested that EndMT was induced by the HG treatment.

HG-induced EndMT expresses mesenchymal stem markers and acquires chondrocyte differentiation potential

To determine whether EndMT could cause an acquisition of MSCs-like phenotype, HAECs were treated with HG to examine the expression of the MSC markers CD44, CD10 and STRO-1. We performed immunofluorescence with antibodies for the MSC markers CD44 and STRO-1. After incubation with $30 \mathrm{mmol} / \mathrm{L} \mathrm{HG}$ for $48 \mathrm{~h}$, the cells showed significantly increased expression of CD44 and STRO-1 compared with the control under
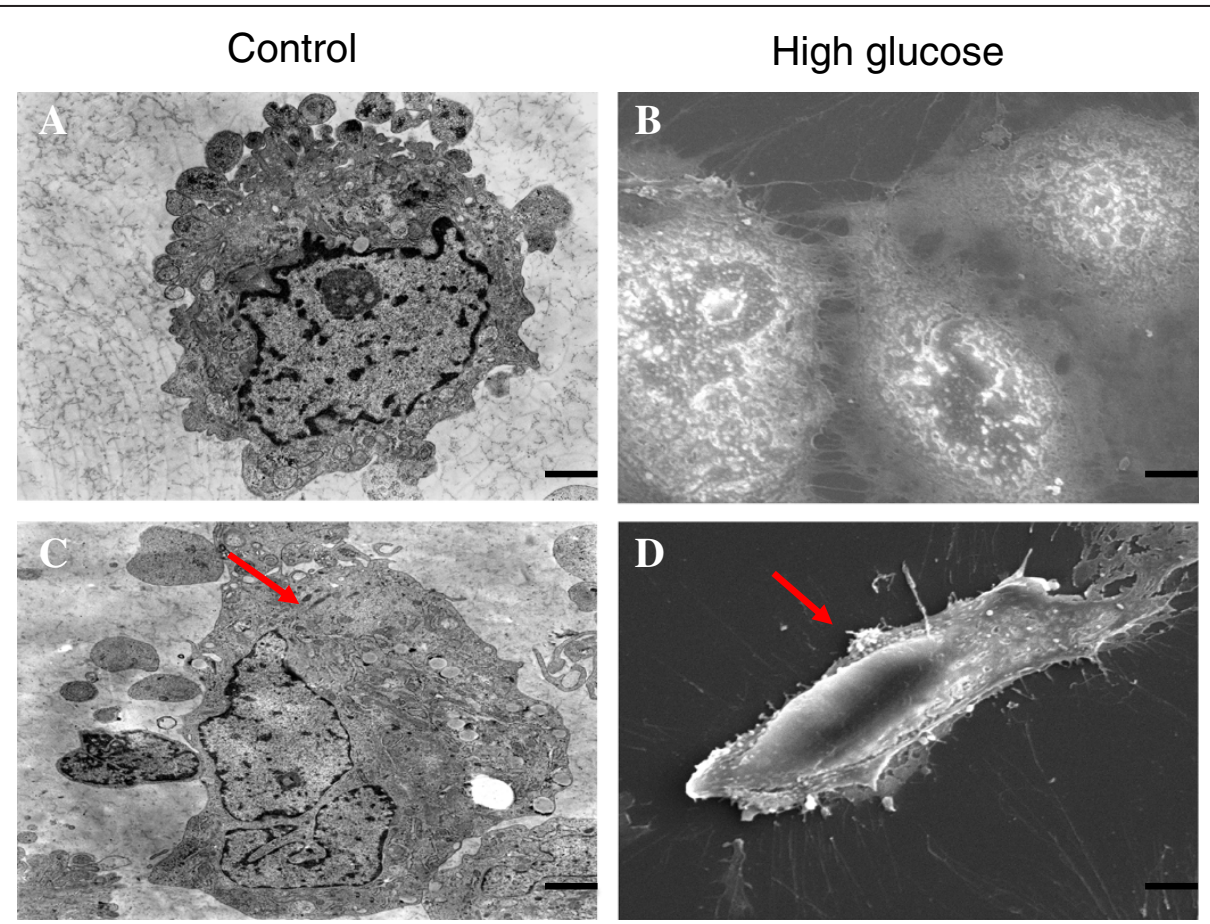

Figure 4 Cellular ultrastructure following HG treatment. Notes: TEM and SEM depicting the change in the cellular ultrastructure following high glucose $(30 \mathrm{mmol} / \mathrm{L})$ exposure. The normal HAECs present with few microfilaments and a rough endoplasmic reticulum (A, B). After exposure to $\mathrm{HG}$, microfilamentation and a swollen rough endoplasmic reticulum appeared in the cytoplasm, and the cells became elongated and spindle-shaped (C, D). 1 bar $=4 \mu \mathrm{m}$. 
confocal microscopy (Figure 5). Compared with the control group, the HG treatment induced significantly increased mRNA expression in CD44 and CD10 at $48 \mathrm{~h}$ post-treatment (Figure 6). In addition, the CD44 and CD10 protein expression was markedly increased (Figure 7).

Because MSCs are multipotent, we next assessed the chondrocyte differentiation capability of endothelial cells that underwent EndMT. We exposed the ECs to the $30 \mathrm{mmol} / \mathrm{L} \mathrm{HG}$ for $48 \mathrm{~h}$ after the cells were grown in chondrogenic culture media for one week. Western blot analysis showed that the expression of the chondrocytespecific marker SOX9 was significantly increased compared with the control (Figure 8). Meanwhile, the HGtreated HAECs stained positively for cartilage proteoglycan alcian blue after growth in chondrogenic medium for 7 days (Figure 9). Consistent with the evaluation of SOX9 expression, calcium deposits as shown by alizarin red staining were also enhanced by HG treatment (Figure 9).
HG-induced EndMT is associated with the activation of the Snail signaling pathway

To determine whether the EndMT triggered by HG observed in our experiments was accompanied by the activation of Snail, a western blot was performed using HAECs treated with or without HG. The protein level of Snail was significantly increased by treatment with HG compared with the control (Figure 10).

\section{Discussion}

Vascular calcification is more common in patients with diabetes compared with the general population and is associated with increased mortality, stroke and amputations [1-3]. In addition, under HG conditions with increased TNF-alpha levels, the death receptors TNF-R1 and Fas, are up-regulated in human coronary artery endothelial cells, which could in turn play a role in HGinduced endothelial cell apoptosis and hyperglycemia appears to be a risk factor for vascular calcification [14-16]. Many studies have shown that HG may cause

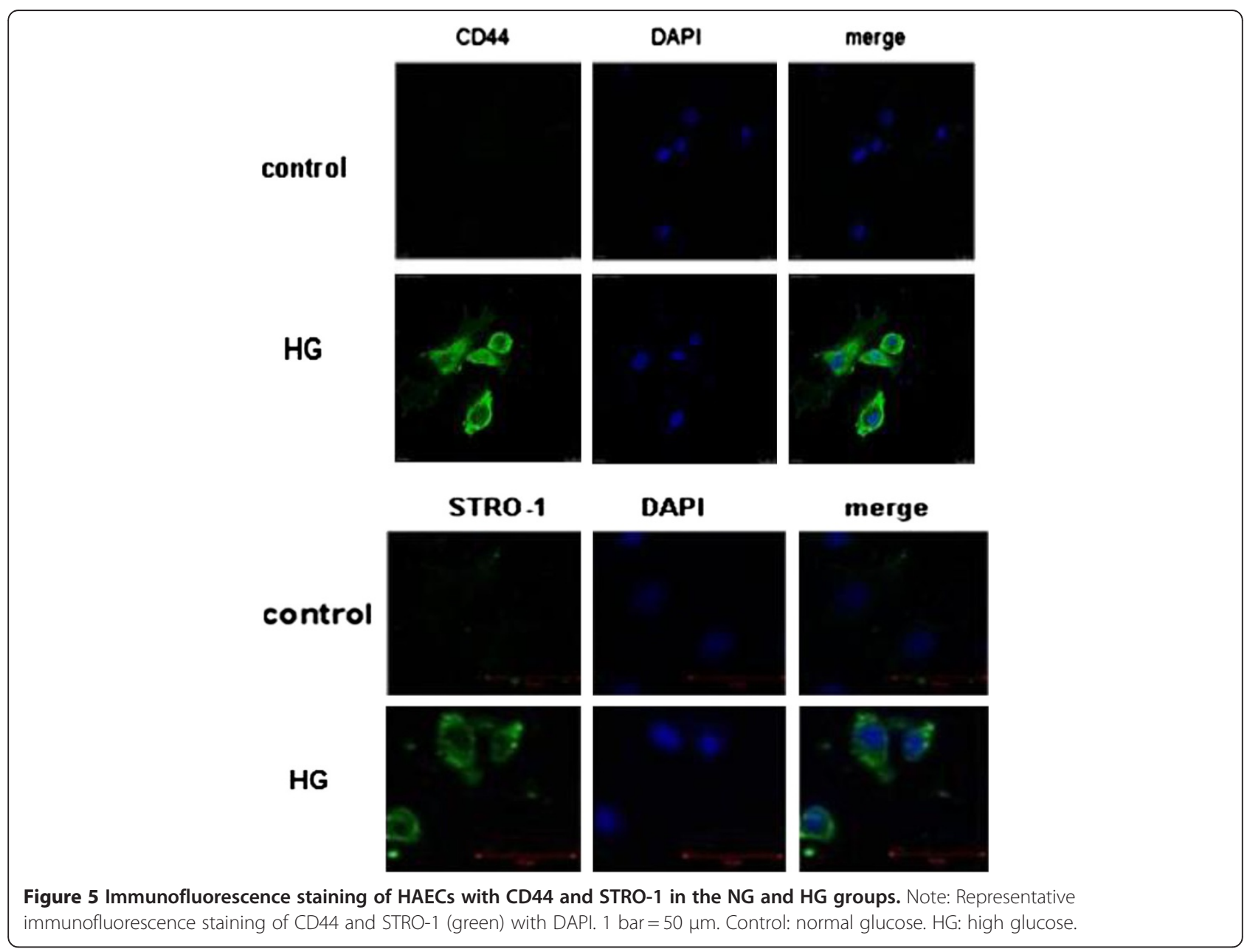




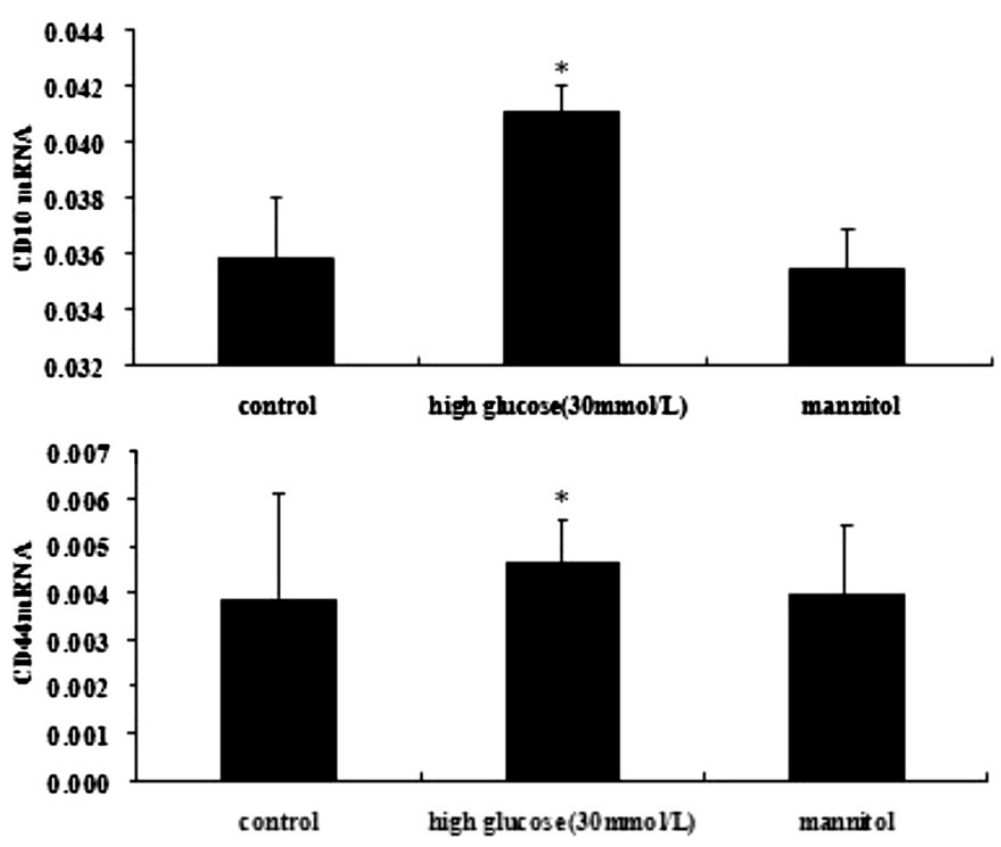

Figure 6 RT-PCR expression of CD10 and CD44 in the NG and HG groups. Notes: HAECs were treated with 30 mmol/L high glucose for the indicated times. The expression of MSCs markers (CD44, CD10) was detected by real-time PCR. The values represent the means $\pm S D .{ }^{*}, P<0.05$ vs. control.

cardiovascular medial calcification and increased levels of plasma Matrix GLA protein indicates the progressing calcification process in patients with type 2 DM [17], but its underlying mechanism is not fully understood in HG-induced endothelial injury. We repeated the same results as a previous study: it showed that the treatment of HAECs with HG led to a significantly increased expression of the FSP1 protein in a dose- and timedependent manner (data not shown). Double staining of the HAECs showed co-localization of CD31 and FSP1, the acquisition of a spindle-shaped morphology by some cells and loss of CD31 staining, which indicated the occurrence of EndMT [18,19]. Furthermore, in this study, the cells undergoing EndMT showed expression of STRO-1, CD44 and SOX9 compared with the controls. Additionally, alcian blue staining in the HG group was positive compared with the NG group. The EndMT resulted in the acquisition of MSCs-like properties to enable the differentiation into chondrocytes, which is in accordance with the report from Medici and colleagues [12]. We first found that the HG-induced EndMT could trigger the conversion into chondrocytes, which are involved in the vascular medial calcification.

Chondrogenesis is a key pathologic mechanism underlying medial calcification $[4,13]$. Given its central role, it is not surprising that the origins of ectopically accumulated chondrocytes in the arterial wall have become an attractive field in research. During the past decade, the notion that SMCs undergo differentiation into chondrogenic cells to participate in MAC has already gained acceptance [5,7]. However, conventional distinctions among vascular cell lineages are becoming less clear as our studies and other investigators determine that ECs could also trans-differentiate into SMCs, a process known as EndMT $[10,11,18,19]$. Furthermore, studies found that EndMT has the ability to convert cells into MSCs and then differentiate into chondrocytes [12].

MSCs are known to be multipotent cells with cartilage-, adipose-, and bone-forming potential that are widespread in calcified lesions [20-23]. Previous studies have determined that MSCs in adult humans and rodents are derived from bone marrow, cord blood, placenta and adipose tissue [21,23]. Recently, vascular endothelial cells have been proposed to be one precursor of MSCs in certain microenvironments [21,24]. TGF- $\beta 2$ treatment-induced EndMT contributes to the acquisition of the MSC phenotype in ECs [12,23]. Kissa et al. [25] indicated that ECs from the aortic ventral wall can transformation into hematopoietic cells in animals. Slukvin et al. [24] showed the MSCs could derive from EndMT. More interestingly, Medici et al. found that chondrocytes at the sites of heterotopic ossification stain positively with antibodies specific for endothelial markers in progressive models of fibrodysplasia ossificans [12]. In our experiment, we found that elevated HG could induce the significantly increased expression of CD44, CD10, and STRO-1 (markers of MSCs) and SOX9 (a transcription factor required for chondrocyte differentiation) in 


\section{CD44}

CD10

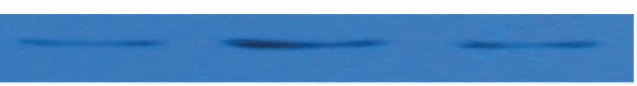

$\beta$-actin
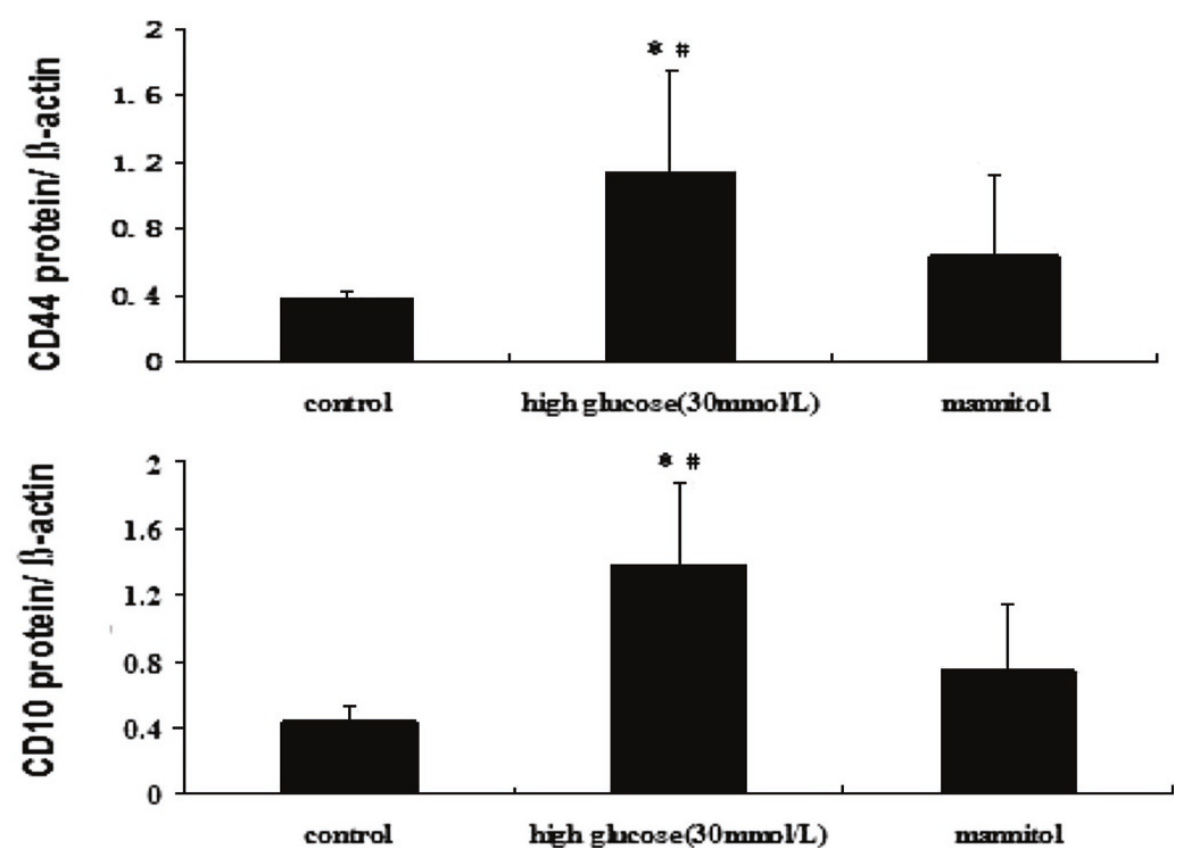

Figure 7 Western blot expression of CD44 and CD10 in the NG and HG groups. Notes: HAECs were treated with or without $30 \mathrm{mmol} / \mathrm{L}$ HG for 48 h. MSCs marker (CD44, CD10) expressions were detected by western blot. $\beta$-actin was used as internal control. Values represent the means \pm SD. ${ }^{*} P<0.05$ vs. control. ${ }^{\#} P<0.05$ vs. mannitol.

\section{SOX9 \\ $\beta$-actin}

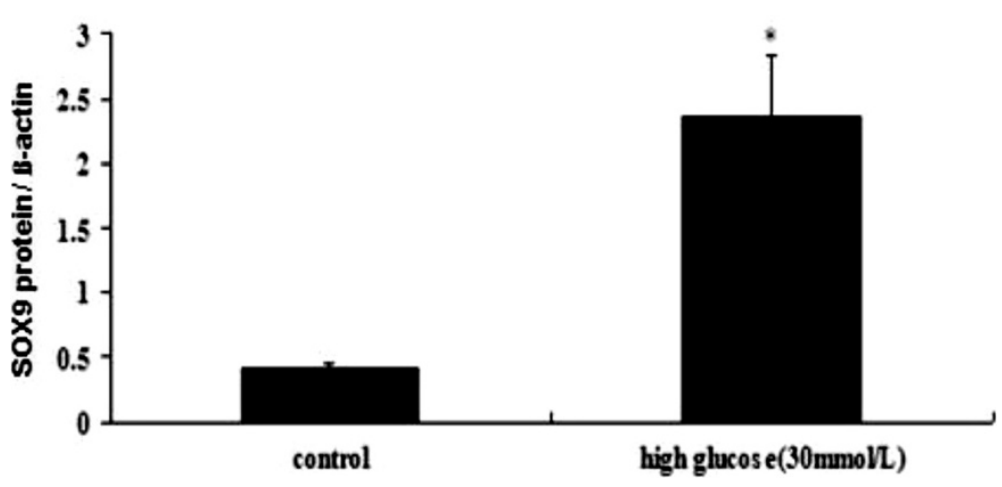

Figure 8 Western blot expression of SOX9 in the NG and HG groups. Notes: Western blot analysis for the chondrocyte marker is shown (SOX9). $\beta$-actin was used as an internal control. The values represent the means \pm SD. ${ }^{*}, P<0.05$ vs. control. 


\section{Control}
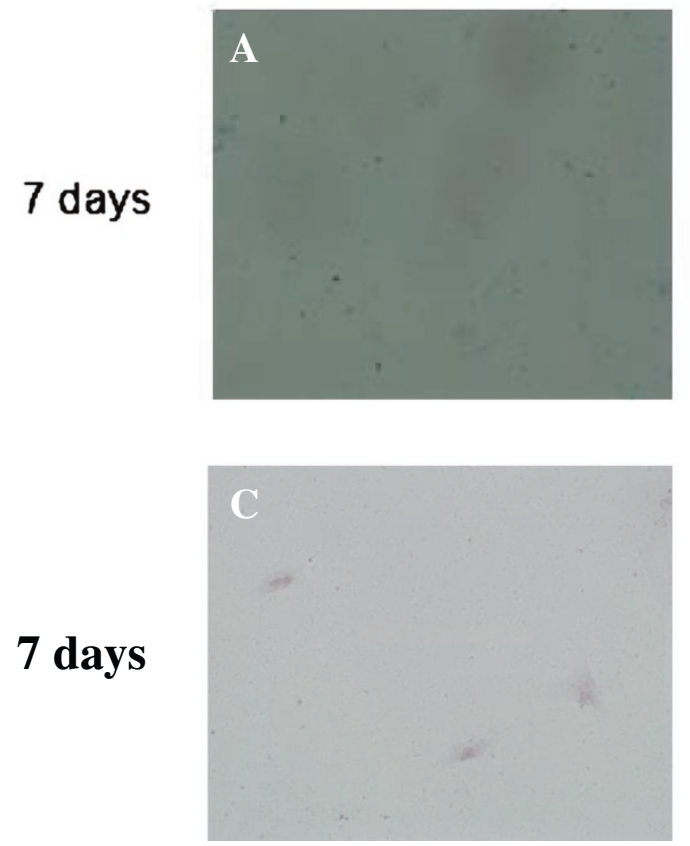

High glucose
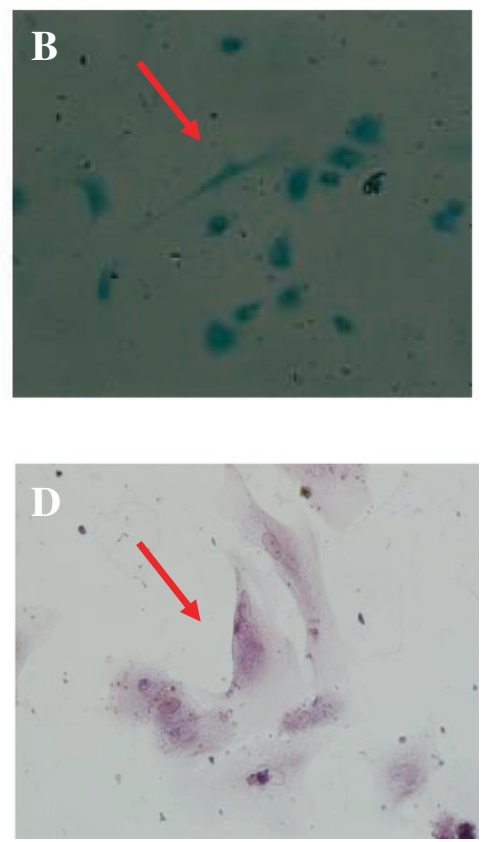

Figure 9 HAECs incubated with HG exhibit chondrocyte differentiation potency. Notes: HAECs were treated with or without 30 mM HG for $48 \mathrm{~h}$ followed by exposure to chondrogenic culture medium for 7 days (A). Alcian blue staining for cells grown in chondrogenic culture medium for 7 days is shown $(\mathbf{B}, \times 100)$. (C). Alizarin Red staining for calcium deposition $(\mathbf{D}, \times 200)$.

cells undergoing EndMT. Meanwhile, HG-treated HAECs stained positively for cartilage proteoglycan alcian blue after growth in chondrogenic medium for 7 days. Consistent with the evaluation of SOX9 expression, calcium deposits as shown by alizarin red staining were also enhanced by HG treatment. All of these experiments suggested that the endothelium may contribute to chondrocyte genesis by MSCs generation, which was involved in the diabetic vascular medial calcification.

Recent work has shown that the exposure of ECs to HG activates several signal transduction networks

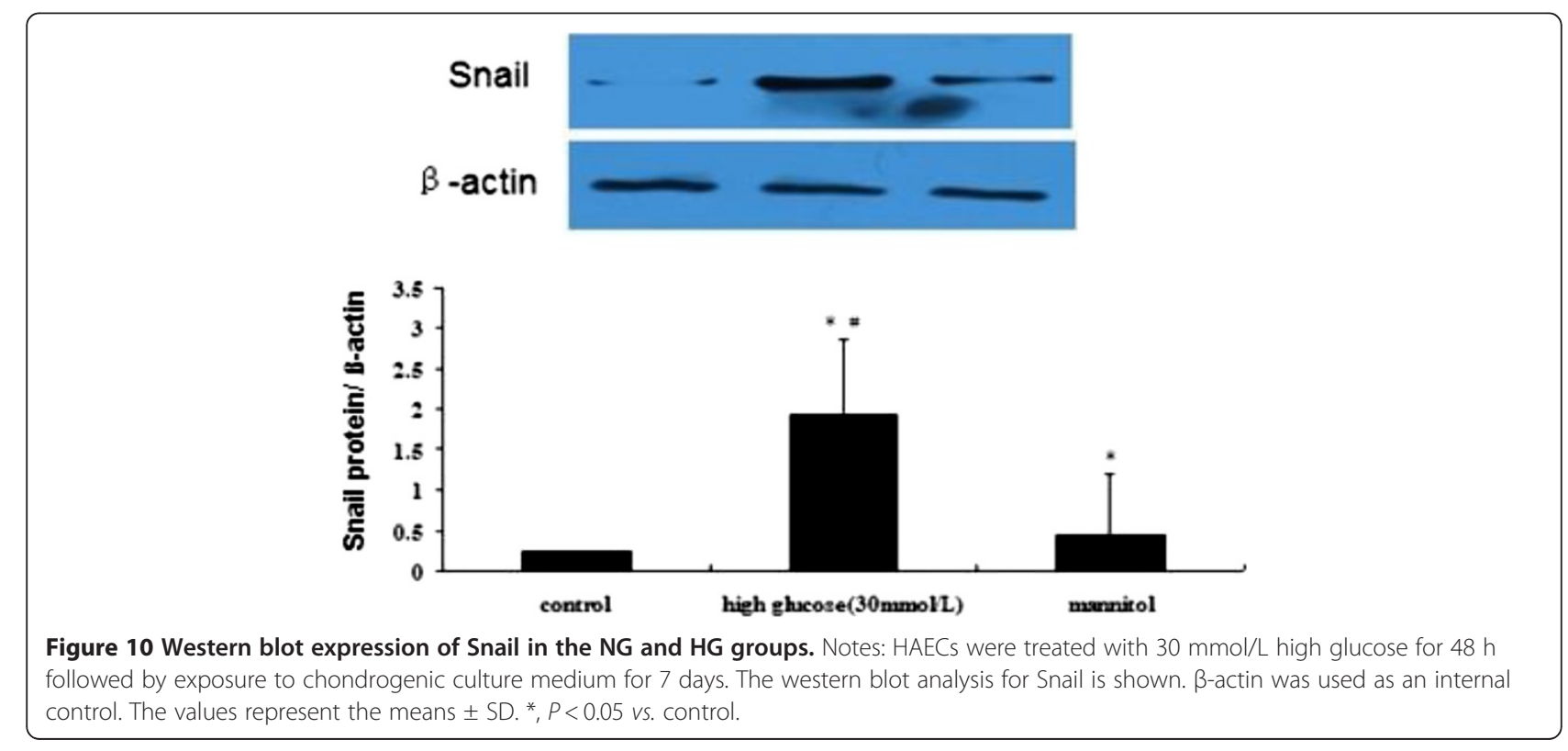


responsible for mediating the proliferative and growthpromoting responses. Snail has been implicated in a wide variety of cellular responses, including transition, growth, gene expression, angiogenesis, contractility and vesicle trafficking. Kokudo et al. [26] found that Snail is required for the TGF $\beta$-induced EndMT of embryonic stem cell-derived ECs. Moreover, TGF- $\beta 2$ promotes Snail-mediated endothelial-mesenchymal transition through the convergence of Smad-dependent and Smad-independent signaling [27]. In addition, a role of the TGF- $\beta$ in the regulation of terminal chondrocyte differentiation was recently reported [28]. Jayachandran and colleagues [29] found that Snail transcription factors mediate the epithelial-mesenchymal transition (EMT) in lung fibrosis. EndMT is known to be a form of EMT that is present during the embryonic development of the heart [30], and glycogen synthase kinase-3 is an endogenous inhibitor of Snail transcription, which has been implicated in EMT [31]. Thus, we addressed the question of whether Snail mediated the HG-induced EndMT. Our results indicated that the protein level of Snail was significantly increased by the HG treatment compared with the control. Therefore, all of these findings support the hypothesis that the effect of HG on EC transdifferentiation into chondrocyte-like cells is mediated, at least in part, through the Snail signaling pathway.

\section{Conclusions}

Our study demonstrated that HG could induce endothelial cells transdifferentiation into chondrocyte-like cells via EndMT, which is mediated in part by the activation of the snail signaling pathway. Understanding the mechanisms that control endothelium transdifferentiation to osteochondroprogenitors and the subsequent vascular calcification may help develop novel strategies that prevent or reverse vascular calcification.

\section{Abbreviations \\ EndMT: Endothelial-to-mesenchymal transition; DM: Diabetes mellitus; FSP- 1: Fibroblast-specific protein1; HAECs: Human aortic endothelial cells; HG: High glucose; MSCs: Mesenchymal stem cells; MA: Cmedial artery calcification; NG: Normal glucose; SMCs: Smooth muscle cells.}

\section{Competing interests}

The authors declare that they have no competing interests.

\section{Authors' contributions}

TR: conception and design, data interpretation, manuscript writing and final approval of manuscript; WM: conception and design, data analysis, manuscript writing, final approval of manuscript; LH and GM: advice with protein assay and data interpretation; LB: conception and design, assembly of data and data interpretation, manuscript review, final approval of manuscript. All authors read and approved the final manuscript.

\section{Authors' information}

Min Gao as Co-first author

Min Wu as Co-corresponding author.

\section{Acknowledgement}

This work was supported by grants from National Natural Science Foundation of China (Key Program, No. 81130010); Natural Science Foundation of Jiangsu Province (No. BK 2011603 and BK 2012751) and Foundation of Southeast University (No. seucx 201107).

Received: 30 July 2012 Accepted: 13 September 2012 Published: 22 September 2012

\section{References}

1. Saleh N, Petursson $P$, Lagerqvist B, Skúladóttir H, Svensson A, Eliasson B, Gudbjörnsdottir S, Eeg-Olofsson K, Norhammar A: Long-term mortality in patients with type 2 diabetes undergoing coronary angiography: the impact of glucose-lowering treatment. Diabetologia 2012, 55:2109-2117.

2. Rennenberg RJ, Kessels AG, Schurgers $L$, van Engelshoven JM, de LeeuW PW, Kroon AA: Vascular calcifications as a marker of increased cardiovascular risk: a meta-analysis. Vasc Health Risk Manag 2009, 5:185-197.

3. Patel T, Charytan DM: Cardiovascular complications in diabetic kidney disease. Semin Dial 2010, 23:169-177.

4. Neven E, Dauwe S, De Broe ME, D'Haese PC, Persy V: Endochondral bone formation is involved in media calcification in rats and in men. Kidney Int 2007, 72:574-581.

5. Sage AP, Tintut $Y$, Demer LL: Regulatory mechanisms in vascular calcification. Nat Rev Cardiol 2010, 7:528-536.

6. Farrington-Rock C, Crofts NJ, Doherty MJ, Ashton BA, Griffin-Jones C, Canfield AE: Chondrogenic and adipogenic potential of microvascular pericytes. Circulation 2004, 110:2226-2232.

7. Speer MY, Yang HY, Brabb T, Leaf E, Look A, Lin WL, Frutkin A, Dichek D, Giachelli CM: Smooth muscle cells give rise ot osteochondrogenic precursors and chondrocyte in calcifying arteries. Circ Res 2009, 104:733-741.

8. Frid MG, Kale VA, Stenmark KR: Mature vascular endothelium can give rise to smooth muscle cells via endothelial-mesenchymal transdifferentiation: in vitro analysis. Circ Res 2002, 90:1189-1196.

9. Zeisberg EM, Potenta SE, Sugimoto H, Zeisberg M, Kalluri R: Fibroblasts in kidney fibrosis emerge via endothelial-to-mesenchymal transition. J Am Soc Nephrol 2008, 19:2282-2287.

10. Li J, Qu X, Yao J, Caruana G, Ricardo SD, Yamamoto Y, Yamamoto H, Bertram JF: Blockade of endothelial-mesenchymal transition by a Smad3 inhibitor delays the early development of streptozotocin-induced diabetic nephropathy. Diabetes 2010, 59:2612-2624.

11. Widyantoro B, Emoto N, Nakayama K, Anggrahini DW, Adiarto S, Iwasa N Yagi K, Miyagawa K, Rikitake Y, Suzuki T, Kisanuki YY, Yanagisawa M, Hirata K: Endothelial cell-derived endothelin-1 promotes cardiac fibrosis in diabetic hearts through stimulation of endothelial-to-mesenchymal transition. Circulation 2010, 121:2407-2418.

12. Medici D, Shore EM, Lounev WY, Kaplan FS, Kalluri R, Olsen BR: Conversion of vascualr endothelial cells into multipotent stem-like cells. Nat Med 2010, 16:1400-1406.

13. Neven E, Persy V, Dauwe S, De Schutter T, De Broe ME, D'Haese PC: Chondrocyte rather than osteoblast conversion of vascular cells underlies medial calcification in uremic rats. Arterioscler Thromb Vasc Biol 2010, 30:1741-1750.

14. Chen NX, Duan D, O'Neill KD, Moe SM: High glucose increases the expression of $\mathrm{Cbfa} 1$ and BMP-2 and enhances the calcification of vascular smooth muscle cells. Nephrol Dial Transplant 2006, 21:3435-3442.

15. Boström Kl, Jumabay M, Matveyenko A, Nicholas SB, Yao Y: Activation of vascular bone morphogenetic protein signaling in diabetes mellitus. Circ Res 2011, 108:446-457

16. Kageyama S, Yokoo H, Tomita K, Kageyama-Yahara N, Uchimido R, Matsuda N, Yamamoto S, Hattori Y: High glucose-induced apoptosis in human coronary artery endothelial cells involves up-regulation of death receptors. Cardiovasc Diabetol 2011, 4:73.

17. Thomsen SB, Rathcke CN, Zerahn B, Vestergaard H: Increased levels of the calcification marker matrix Gla Protein and the inflammatory markers YKL-40 and CRP in patients with type 2 diabetes and ischemic heart disease. Cardiovasc Diabetol 2010, 9:86. 
18. Tang R, Li Q, Lv L, Dai H, Zheng M, Ma K, Liu B: Angiotensin II mediates the high-glucose-induced endothelial-to-mesenchymal transition in human aortic endothelial cells. Cardiovasc Diabetol 2010, 9:31.

19. Tang RN, Lv LL, Zhang JD, Dai HY, Li Q, Zheng M, Ni J, Ma KL, Liu BC: Effect of angiotensis II receptor blocker on myocardial endothelial-tomesenchymal transition in diabetic rats. Int J Cardio 2011, in press.

20. Moe SM, Chen NX: Mechanisms of vascular calcification in chronic kidney disease. J Am Soc Nephrol 2008, 19:213-216.

21. Horwitz EM: Building bone from blood vessels. Nat Med 2010, 16:1373-1374

22. Medici D, Kalluri R: Endothelial-mesenchymal transition and its contribution to the emergence of stem cell phenotype. Semin Cancer Biol 2012, in press.

23. Shoshani O, Zipori D: Transition of endothelium to cartilage and bone. Cell Stem Cell 2011, 8:10-11.

24. Slukvin II, Vodyanik M: Endothelial origin of mesenchymal stem cells. Cell Cycle 2011, 10:1370-1373.

25. Kissa K, Herbomel P: Blood stem cells emerge from aortic endothelium by a novel type of cell transition. Nature 2010, 464:112-115.

26. Kokudo T, Suzuki Y, Yoshimatsu Y, Yamazaki T, Watabe T, Miyazono K: Snail is required for TGF beta-induced endothelial-mesenchymal transition of embryonic stem cell-derived endothelial cells. J Cell Sci 2008, 121:3317-3324.

27. Medici D, Potenta S, Kalluri R: Transforming growth factor- $\beta 2$ promotes Snail-mediated endothelial-mesenchymal transition through convergence of Smad-dependent and Smad-independent signalling. Biochem J 2011, 437:515-520.

28. Sueyoshi T, Yamamoto K, Akiyama H: Conditional deletion of Tgfbr2 in hypertrophic chondrocytes delays terminal chondrocyte differentiation. Matrix Biol 2012, in press.

29. Jayachandran A, Königshoff M, Yu H, Rupniewska E, Hecker M, Klepetko W, Seeger W, Eickelberg O: SNAI transcription factors mediate epithelialmesenchymal transition in lung fibrosis. Thorax 2009, 64:1053-1061.

30. Zeisberg EM, Tarnavski O, Zeisberg M, Dorfman AL, McMullen JR, Gustafsson E, Chandraker A, Yuan X, Pu WT, Roberts AB, Neilson EG, Sayegh MH, Izumo S, Kalluri R: Endothelial-to-mesenchymal transition contributes to cardiac fibrosis. Nat Med 2007, 13:952-961.

31. Bachelder RE, Yoon SO, Franci C, de Herreros AG, Mercurio AM: Glycogen synthase kinase- 3 is an endogenous inhibitor of Snail transcription: implications for the epithelial-mesenchymal transition. J Cell Biol 2005, 168:29-33.

doi:10.1186/1475-2840-11-113

Cite this article as: Tang et al: High glucose mediates endothelial-tochondrocyte transition in human aortic endothelial cells. Cardiovascular Diabetology 2012 11:113.

\section{Submit your next manuscript to BioMed Central and take full advantage of:}

- Convenient online submission

- Thorough peer review

- No space constraints or color figure charges

- Immediate publication on acceptance

- Inclusion in PubMed, CAS, Scopus and Google Scholar

- Research which is freely available for redistribution 